\title{
A Universal Correlation between the Duration and the X-ray Luminosity in Stellar Flares
}

\author{
Y. Tsuboi*, R. Sasaki \\ Department of Physics, Faculty of Science \& Engineering, Chuo University, 1-13-27 Kasuga, \\ Bunkyo, Tokyo 112-8551, Japan \\ E-mail: tsuboi@phys.chuo-u.ac.jp, sasakiephys.chuo-u.ac.jp
}

\section{Y. Sugawara}

Institute of Space and Astronautical Science, Japan Aerospace Exploration Agency, 3-1-1

Yoshinodai, Sagamihara, Kanagawa 252-5210, Japan

E-mail: sugawara.yasuharu@jaxa.jp

\section{Matsuoka}

MAXI team, Institute of Physical and Chemical Research (RIKEN), 2-1 Hirosawa, Wako,

Saitama 351-0198, Japan

E-mail: matsuoka.masarueriken.jp

\begin{abstract}
Since the launch in 2009 August, with the unprecedentedly high sensitivity as an all-sky X-ray monitor, MAXI has caught more than a hundred of huge flares from stars. Most of them are from low-mass, active stars (RS CVn systems, an Algol system, dMe systems, a dKe system, Young Stellar Objects). With the total radiative energy of $10^{34}-10^{39}$ ergs, the MAXI detections have broken the record of the largest flaring magnitudes in each stellar categories. The enlarged sample of intense flares has enabled us to do systematic studies in various viewpoints. One of the studies is the discovery of a universal correlation between the flare duration and the intrinsic Xray luminosity, which holds for 5 and 12 orders of magnitude in the duration and $\mathrm{L}_{\mathrm{X}}$, respectively (Tsuboi et al. 2016). Here, we review the studies of stellar flares obtained with MAXI.
\end{abstract}

XII Multifrequency Behaviour of High Energy Cosmic Sources Workshop

12-17 June, 2017

Palermo, Italy

${ }^{*}$ Speaker. 


\section{Introduction}

X-ray flares are observed on cool stars including the Sun. The flares are characterized with the fast-rise and slow-decay light curve. Those rapid time-variability generally follows the rise and decay in the plasma temperature. The general understanding, based on the numerous studies of solar flares, is that such features arise as a consequence of a sudden energy release and relaxation process in the reconnection of magnetic fields on/around stellar surfaces.

The study of the X-ray flares have been made with pointing observations ( [9], [4], [1], [3], and references therein) and monitoring observations with X-ray all-sky monitors such as Ariel-V/SSI, GRANAT/WATCH, and Swift/BAT ([10] and [11]). Using the data of Ariel-V/SSI spanning for 5.5 years, Pye et al. (1983) and Rao et al. (1987) detected in total twenty flares from seventeen stellar sources, including ten RS CVn systems and seven dMe stars ([10], [11]). Rao et al. (1987) showed that there is a positive correlation between the bolometric luminosity and the X-ray peak luminosity.

Recently, an intensive monitoring of large flares with all-sky X-ray surveys, has been made with the Monitor of All-sky X-ray Image (MAXI; [5]). The number of the detection of the large flare was increased significantly. Tsuboi et al. (2016) found the correlation between X-ray bolometric luminosity and the duration time which holds from solar microflares to giant stellar flares detected with MAXI [15].

In this paper, we review the giant flare samples obtained with MAXI, and the derived correlation between the duration of and $\mathrm{L}_{X}$ in stellar flares. MAXI is a mission of an all-sky X-ray monitor operated in the Japanese Experiment Module (JEM; Kibo) on the International Space Station (ISS) since 2009 August. It observes an area in the sky once per $92 \mathrm{~min}$ orbital cycle, and enables us to search for stellar flares effectively.

\section{MAXI Monitoring Observations}

The MAXI monitoring observations of the stellar flares have been conducted with the GSC detector. The detector covers an energy range of 2 to $30 \mathrm{keV}[5,6]$. The data from 2009 August 15th to 2011 August 15th are used there. All the data they used were delivered from the MAXI database system [7].

The GSC consists of twelve pieces of proportional counters, which employ resistive carbonwire anodes to acquire one-dimensional position sensitivity. The GSC typically scans a point source on the sky during a transit of 40-150 seconds with a FoV of $1^{\circ} .5$-width (FWHM) every 92-minute orbital period. The transit time depends on the source incident angle in the Anode-Wire Direction. The detector area for the target changes according to the triangular transmission function of the collimator during each transit. The peak value is $4-5 \mathrm{~cm}^{2}$ per one camera. The detailed performance of the GSC was described by [14].

\section{MAXI Flare Sample}

During the two-year MAXI/GSC survey, Tsuboi et al. (2016) detected twenty-three energetic flares from thirteen active stars (eight RS-CVn stars, three dMe stars, one YSO, and one Algol 
type star) [15]. The physical parameters of the flares are very large for stellar flares in all of the followings: the X-ray luminosity $L_{\mathrm{X}}\left(10^{31-34} \mathrm{ergs} \mathrm{s}^{-1}\right.$ in the $2-20 \mathrm{keV}$ band), the emission measure EM $\left(10^{54-57} \mathrm{~cm}^{-3}\right)$, the $e$-folding time $\left(10^{3-6} \mathrm{~s}\right)$, and the total energy released during the flare $\left(10^{34-39} \mathrm{ergs}\right)$.

The survey showed that the number of the flare sources with extremely large flares is very limited; only ten out of the 256 active binaries within the $100 \mathrm{pc}$ distance have been detected, while four of the ten sources showed flares multiple times. The MAXI stellar sample has especially fast rotation velocities with an order of $10 \mathrm{~km} \mathrm{~s}^{-1}$. This indicates that the rotation velocity is an essential parameter to generate big flares.

They detected no X-ray flares from solar-type stars, despite the fact that fifteen G-type mainsequence stars lie within 10-pc distance. This implies that the frequency of the superflares from solar-type stars, which has $L_{X}$ of more than $1 \times 10^{30} \mathrm{ergs} \mathrm{s}^{-1}$, is very small.

On the $E M-k T$ plot, their sample is located at the high ends in the universal correlation, which ranges over orders of magnitude ([2], [12]). According to the theory of Shibata \& Yokoyama (1999) [12], the MAXI sample has the similar intensity of magnetic field to those detected on the Sun $(\sim 15-150 \mathrm{G})$, but has orders of magnitude larger flare-loop sizes than those on the Sun $(<0.1$ $\left.R_{\odot}\right)$. The largest two loop sizes from UX Ari and II Peg are huge, and might be much larger than even the binary separations.

\section{A Universal Correlation between the Duration and the X-ray Luminosity}

Tsuboi et al. (2016) plotted the duration vs. the intrinsic X-ray luminosity ( $\left.L_{\mathrm{X} \_ \text {bol }}\right)$ in the 0.1-100 keV band, using the data of solar and stellar flares in literatures and the data of the flares on MAXI/GSC sources (see Fig. 1). The plot indicates that there is a universal positive correlation between $L_{\mathrm{X} \_ \text {bol }}$ of a flare and its duration, such that a longer duration means a higher $L_{\mathrm{X} \_ \text {bol }}$. The correlation holds for the wide range of parameter values; 12 and 5 orders of magnitude in $L_{\mathrm{X} \_ \text {bol }}$ and duration, respectively. The MAXI sample is located at the highest ends on the correlation. From the data, they found that the duration is proportional to $L_{\mathrm{X}_{\mathrm{B}} \mathrm{ol}}^{0.2}$.

The positive correlation between the flare duration and the $\bar{X}$-ray luminosity can be described with the cooling model of a single flare. The cooling can be modeled by either a radiative or conductive process. More luminous flare has higher temperature, as we see in the $E M-k T$ diagram (see above section). In the radiative cooling model, simply, the higher temperature makes the cooling time longer. In the conductive cooling model, it is expected that the more luminous flare accompanies with the longer flare loop. The longer flare loop makes the conductive cooling timescale longer.

\section{References}

[1] Favata, F. \& Micela, G. 2003, Stellar Coronal Astronomy, Space Science Reviews, 108, 577

[2] Feldman, U., Laming, J. M., \& Doschek, G. A. 1995, The Correlation of Solar Flare Temperature and Emission Measure Extrapolated to the Case of Stellar Flares, The Astrophysical Journal, Letters, 451, L79

[3] Güdel, M. 2004, X-ray astronomy of stellar coronae, Astronomy and Astrophysics Reviews, 12, 71 


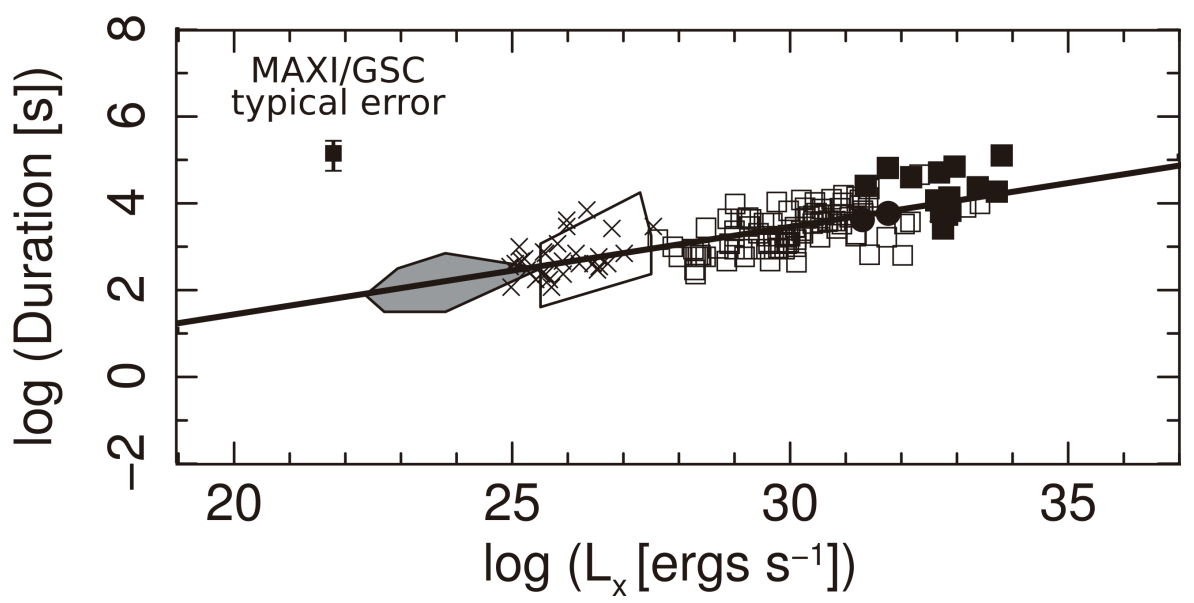

Figure 1: Log-log plot of the duration of flares vs. X-ray luminosity in the $0.1-100 \mathrm{keV}$ band (from Fig. 5 in Tsuboi et al. 2016). The best-fit model is inserted with a broad solid line. The filled squares, filled diamond, filled circles and filled triangle show RS-CVn type stars, Algol, dMe stars and TWA-7, respectively. Stellar flares from RS-CVn type, Algol, dMe stars and YSOs in literatures are indicated with open squares. Three sets of data for solar flares are also superposed: $\mathrm{X}$ marks, large open pentagon and large gray region, taken from [8], [16] and [13], respectively.

[4] Haisch, B., Strong, K., \& Rodono, M. 1991, Flares on the sun and other stars, ARAA, 29, 275

[5] Matsuoka, M., Kawasaki, K., Ueno, S., et al. 2009, The MAXI Mission on the ISS: Science and Instruments for Monitoring All-Sky X-Ray Images, PASJ, 61, 999

[6] Mihara, T., Nakajima, M., Sugizaki, M., et al. 2011, Gas Slit Camera (GSC) onboard MAXI on ISS, PASJ, 63, 623

[7] Negoro, H., Kohama, M., Suzuki, M., et al. 2016, The MAXI/GSC Nova-Alert System and results of its first 68 months, PASJ, 68, S1

[8] Pallavicini, R., Serio, S., \& Vaiana, G. S. 1977, A survey of soft X-ray limb flare images - The relation between their structure in the corona and other physical parameters, The Astrophysical Journal, 216, 108

[9] Pettersen, B. R. 1989, Chromospheric lines in red dwarf flare stars. III, A\&A, 209, 279

[10] Pye, J. P., \& McHardy, I. M. 1983, The Ariel V sky survey of fast-transient X-ray sources, MNRAS, 205,875

[11] Rao, A. R., \& Vahia, M. N. 1987, Fast transient X-rays from flare stars and RS CVn binaries, A\&A, 188, 109

[12] Shibata, K., \& Yokoyama, T. 1999, Origin of the Universal Correlation between the Flare Temperature and the Emission Measure for Solar and Stellar Flares, The Astrophysical Journal, Letters, 526, L49

[13] Shimizu, T. 1995, Energetics and Occurrence Rate of Active-Region Transient Brightenings and Implications for the Heating of the Active-Region Corona, PASJ, 47, 251

[14] Sugizaki, M., Mihara, T., Serino, M., et al. 2011, In-Orbit Performance of MAXI Gas Slit Camera (GSC) on ISS, PASJ, 63, 635 
[15] Tsuboi, Y., Yamazaki, K., Sugawara, Y., et al. 2016, Large X-ray flares on stars detected with MAXI/GSC: A universal correlation between the duration of a flare and its X-ray luminosity, PASJ, 68, 90

[16] Veronig, A., Temmer, M., Hanslmeier, A., Otruba, W., \& Messerotti, M. 2002, Temporal aspects and frequency distributions of solar soft $X$-ray flares, A\&A, 382, 1070 